
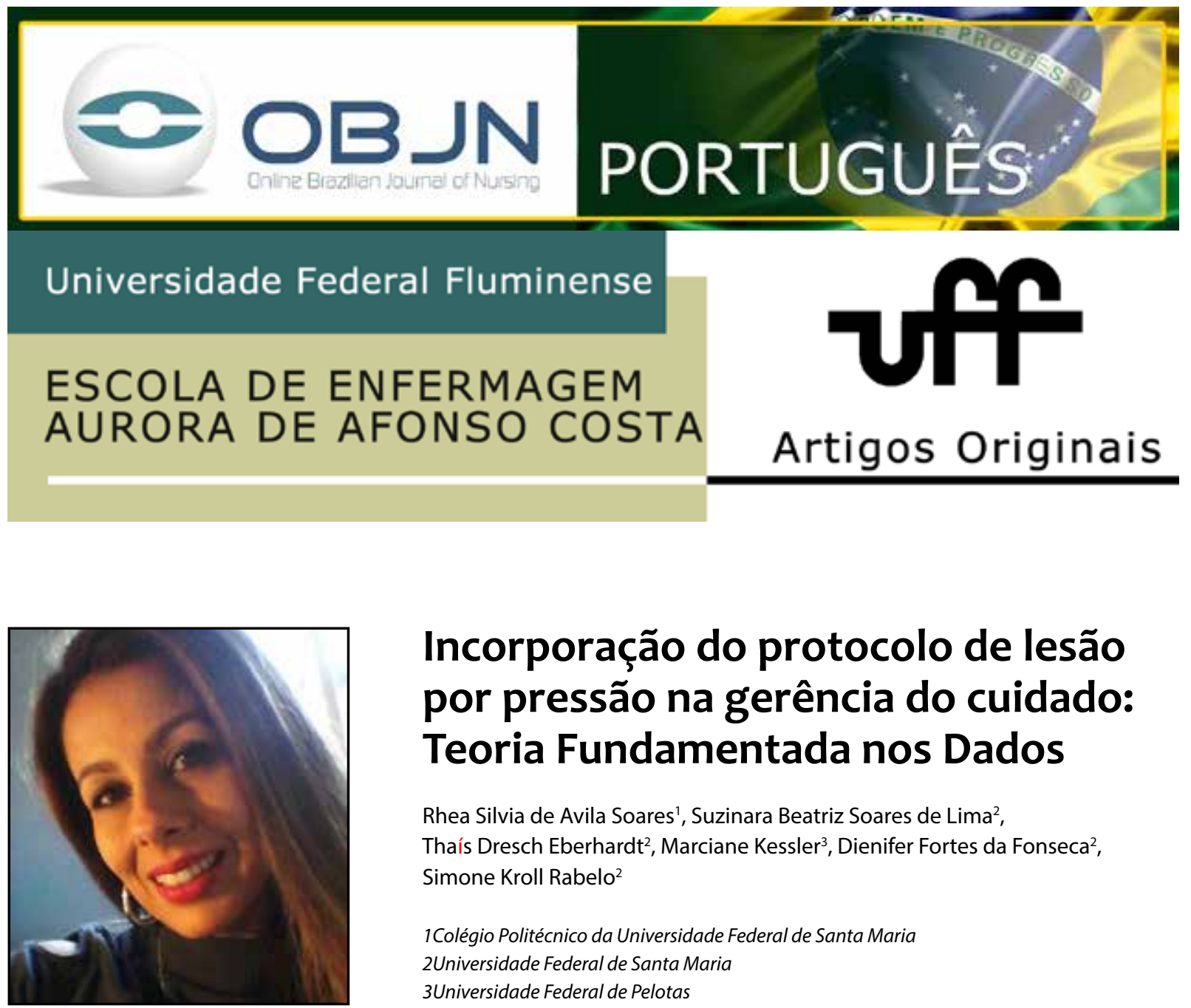

\title{
Incorporação do protocolo de lesão por pressão na gerência do cuidado: Teoria Fundamentada nos Dados
}

\author{
Rhea Silvia de Avila Soares ${ }^{1}$, Suzinara Beatriz Soares de Lima², \\ Simone Kroll Rabelo ${ }^{2}$ \\ 1 Colégio Politécnico da Universidade Federal de Santa Maria \\ ZUniversidade Federal de Santa Maria \\ 3Universidade Federal de Pelotas
}

Thaís Dresch Eberhardt ${ }^{2}$, Marciane Kessler ${ }^{3}$, Dienifer Fortes da Fonseca²,

\section{RESUMO}

Objetivo: Compreender o significado da incorporação do protocolo de lesão por pressão na gerência do cuidado realizada pelo enfermeiro. Método: Pesquisa qualitativa que utilizou referencial metodológico da Teoria Fundamentada nos Dados. A coleta de dados ocorreu por meio de entrevista semiestruturada, com 22 enfermeiros de um hospital universitário, no período de julho a setembro de 2014. Resultados: O fenômeno central "Significando o protocolo de lesão por pressão como instrumento de qualificação do enfermeiro para o cuidado gerencial", composto pela categoria "Qualificando os profissionais para o gerenciamento do cuidado" e suas quatro subcategorias, quais sejam: "Percebendo a qualificação profissional a partir do protocolo", "Considerando o protocolo uma ferramenta de ensino", "Percebendo o protocolo como instrumento de educação em serviço", e "Evidenciando a importância dos protocolos na segurança do paciente". Conclusão: O estudo evidenciou que o protocolo é uma ferramenta de cuidado, bem como um instrumento de educação em serviço, que torna mais seguro o gerenciamento do cuidado e ainda promove a qualificação profissional.

Descritores: Enfermagem; Qualidade da Assistência à Saúde; Protocolos; Lesão por Pressão; Teoria Fundamentada. 


\section{INTRODUÇÃO}

A gestão ou gerência de enfermagem é uma atribuição complexa do enfermeiro, pois envolve relações de causa e efeito por meio de análises científicas de sua prática gerencial(1). Aos enfermeiros cabe o desenvolvimento da assistência de enfermagem, em todos os espaços de atenção à saúde, o que requer competências atreladas ao gerenciamento/administração do cuidado. Além disso, a gerência e a assistência de enfermagem são funções fundamentais no cotidiano desses profissionais e objetivam a qualidade da atenção nas situações de saúde e de doença ${ }^{(2,3)}$

Historicamente, a enfermagem apresenta uma divisão no trabalho feita por Florence Nightingale. Considerada a primeira administradora hospitalar, dividiu as atividades da enfermagem entre nurses e ladies nurses, as quais desenvolvem, respectivamente, atividades gerenciais (indiretas) e assistenciais (diretas) (4). Hoje percebe-se uma aproximação entre gerenciar e assistir, quando estes conceitos se complementam e o gerenciamento do cuidado passa a ser entendido a partir de uma relação dialética entre o saber-fazer do cuidar e do gerenciar $^{(5)}$.

O delineamento gerencial do cuidado deve ser considerado no cotidiano de trabaIho do enfermeiro. Esse, como gerenciador do saber-fazer de forma diferenciada e complexa, necessita estar preparado para a administração de situações de risco, o que implica na tomada de decisões imediatas, tanto na coordenação da equipe, quanto no cuidado prestado aos pacientes em condições críticas ${ }^{(6)}$.

Os enfermeiros, por meio do gerenciamento, implementam estratégias importantes a fim de construir adequadas formas de cuidado, a partir das principais tendências e demandas do seu processo de trabalho. Essas estratégias são sustentadas por importantes ferramentas, entre elas, os protocolos assistenciais que promovem o planejamento do cuidado, os quais são considerados instrumentos de apoio teórico-práticas que contribuem para a qualidade da assistência aos pacientes, pois permitem a sistematização e a padronização da prática de enfermagem ${ }^{(6)}$.

Nesse contexto, destaca-se o gerenciamento do cuidado aos pacientes em risco de desenvolver lesão por pressão (LP). Essas lesões representam um problema de saúde pública que causa impacto sobre a pessoa, a família e a sociedade, além de se constituírem como um indicador de qualidade na saúde ${ }^{(7)}$.

As LP são definidas como danos localizados na pele e/ou no tecido mole subjacente do paciente, que frequentemente ocorrem sobre uma proeminência óssea ou estão relacionadas a equipamentos médicos ou outro tipo de dispositivo. Caracterizam-se como uma lesão na pele intacta ou como uma úlcera aberta e seu desenvolvimento dá-se pela intensidade ou tempo da pressão associada ao cisalhamento. Podem ser afetadas pelo microclima, nutrição, perfusão e doenças associadas ${ }^{(8)}$.

A inovação do processo gerencial do enfermeiro, no que se refere à articulação da gerência e assistência, a partir do estabelecimento de estratégias que impliquem em um cuidado integral e humanizado do paciente na prevenção e no tratamento de LP é cada vez mais instigada. Nesse sentido, recomenda-se o uso de protocolos que, enquanto instrumentos de organização e implementação do cuidado que fazem parte da gerência do processo assistencial, qualificam as ações realizadas pelos profissionais, sustentadas pelas melhores evidências científicas.

No entanto, percebe-se que os enfermeiros não compreendem os protocolos como instrumento de gerência do cuidado. Estudo 
de revisão que avaliou a produção brasileira sobre protocolos de prevenção e tratamento de LP identificou que essa ferramenta reduz a incidência de LP e qualifica o cuidado. Porém, os artigos não apresentam articulações e relações dos protocolos enquanto ferramentas no gerenciamento do cuidado ${ }^{(9)}$, justificando, assim, esta pesquisa.

Com base nessa perspectiva, tem-se a seguinte questão de estudo: qual o significado atribuído pelos enfermeiros, a partir de um protocolo assistencial, sobre a gerência do cuidado de LP? O objetivo delineado foi: compreender o significado da incorporação do protocolo de LP na gerência do cuidado realizada pelo enfermeiro.

\section{MÉTODO}

Pesquisa qualitativa com aporte metodológico da Teoria Fundamentada nos Dados (TFD), a partir da vertente straussiana, método que busca compreender, a partir dos dados extraídos das realidades empíricas, por meio das relações e interações vivenciadas pelos sujeitos, o significado que determinado contexto ou objeto tem para a pessoa, gerando conhecimentos, aumentando a compreensão e proporcionando um guia significativo para a ação(10).

O cenário do estudo foi um hospital universitário, localizado no Sul do Brasil. A coleta de dados se deu por entrevista individual, semiestruturada, gravada em meio digital, realizada na própria instituição, a partir de agendamento com os enfermeiros, no período de julho a setembro de 2014. As entrevistas duraram, em média, 40 minutos. Para sua condução, utilizou-se a seguinte questão norteadora: qual o significado da incorporação do protocolo de LP na gerência do cuidado?
A seleção dos participantes foi intencional, com base no objetivo do estudo, e teve como critérios de inclusão: todos os enfermeiros lotados na Unidade de Terapia Intensiva (UTI) de adultos, na Clínica Médica II e na Unidade Cardíaca Intensiva (UCI), independentemente de seu tempo de serviço. Os critérios de exclusão adotados foram: profissionais em licença, de qualquer natureza, no momento da coleta de dados. Assim, o estudo contou com a participação de 22 enfermeiros.

As entrevistas foram imediatamente transcritas e analisadas após sua realização. Esse processo aconteceu em três etapas interdependentes e complementares: codificação aberta, codificação axial e, por fim, codificação seletiva, que permitiram o refinamento das categorias e subcategorias para definição do fenômeno central ${ }^{(10)}$.

A categoria "Qualificando os profissionais para o gerenciamento do cuidado" é representada como consequência do modelo paradigmático proposto pelo método. Esse modelo é uma estrutura analítica que permite reunir e ordenar os dados sistematicamente, de forma que a estrutura e o processo sejam integrados para revelação do fenômeno central do estudo ${ }^{(10)}$. Ele estabelece a inter-relação entre as subcategorias e as categorias, por meio dos seguintes componentes: fenômeno, contexto, condições causais e intervenientes, estratégias e consequências ${ }^{(10)}$.

Ressalta-se que a pesquisa atendeu aos aspectos éticos da Resolução 466/12 do Conselho Nacional de Saúde, sendo o projeto aprovado por Comitê de Ética em Pesquisa com Seres Humanos, sob o Certificado de Apresentação para Apreciação Ética (CAAE) 30531314.7.0000.5346 e Parecer no 667.777. Visando garantir o anonimato dos participantes, seus nomes foram substituídos por um código composto pela letra " $E$ " seguida do número de ordem da entrevista (E1, E2, E3... E22). 


\section{RESULTADOS}

Do fenômeno "Significando o protocolo de lesão por pressão como instrumento de qualificação do enfermeiro para o cuidado gerencial"12, apresentar-se-ão as consequências compostas pela categoria "Qualificando os profissionais para o gerenciamento do cuidado" e suas quatro subcategorias: "Percebendo a qualificação profissional a partir do protocolo", "Considerando o protocolo uma ferramenta de ensino", "Percebendo o protocolo como instrumento de educação em serviço", e "Evidenciando a importância dos protocolos na segurança do paciente". O diagrama representativo da categoria e suas subcategorias está representado na Figura 1.
PERCEBENDO A QUALIFICAÇÃO PROFISSIONAL A PARTIR DO PROTOCOLO

Os enfermeiros atribuíram ao protocolo o desenvolvimento de um gerenciamento do cuidado com qualidade, uma vez que sua utilização implica em melhores práticas no processo de trabalho. Garante-se, assim, um cuidado inovador e sistematizado, com fundamentos teóricos para promoção da saúde, além de permitir a redução da variabilidade de ações. Pode-se observar essa constatação através dos trechos abaixo, extraídos das entrevistas realizadas com os enfermeiros:

A qualidade melhorou com o protocolo, não só o de lesão por pressão,

Figura 1. Diagrama representativo do componente "consequência" do fenômeno "Significando o protocolo de lesão por pressão como instrumento de qualificação do enfermeiro para o cuidado gerencial"

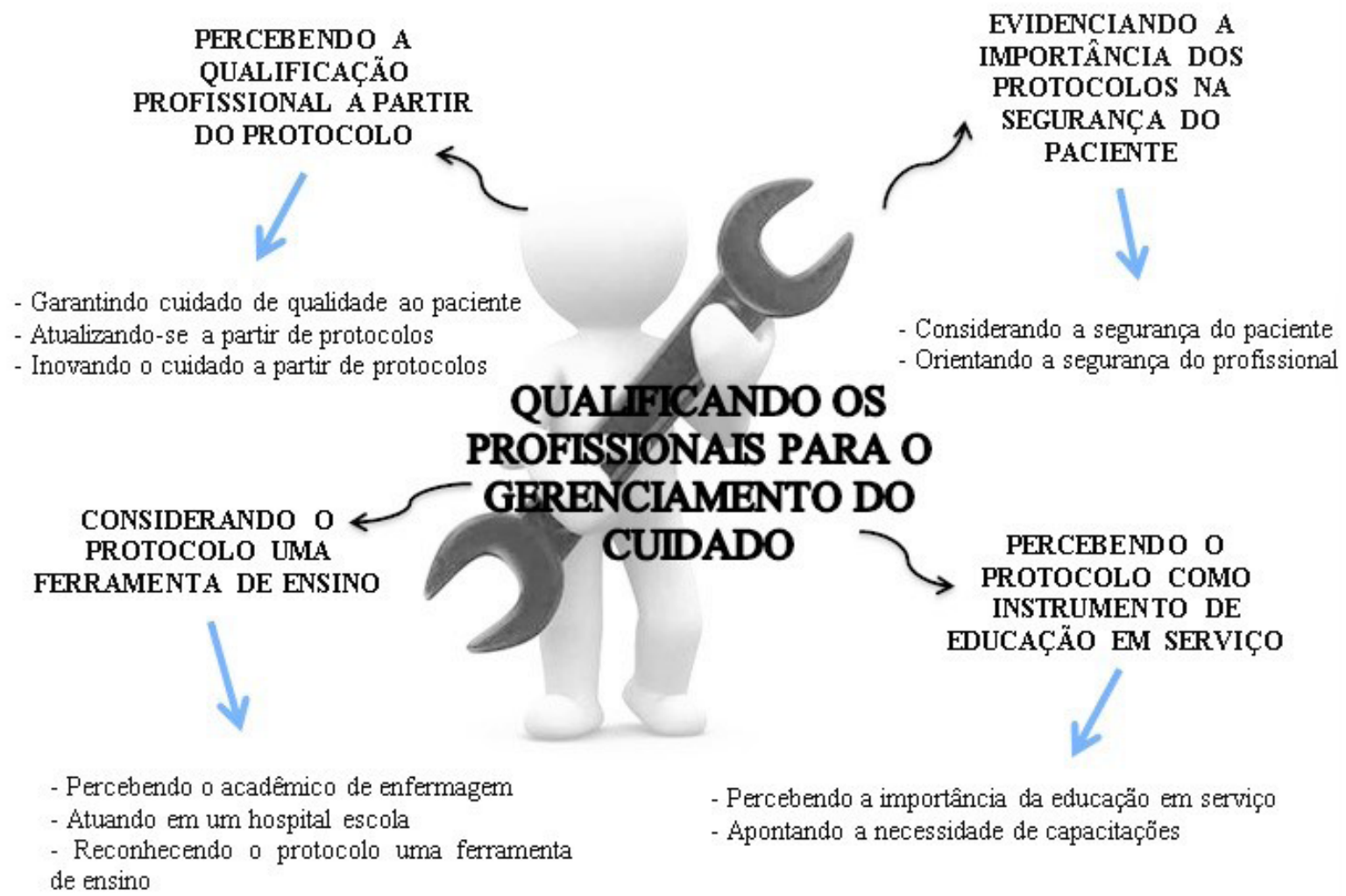

Fonte: Dissertação de Mestrado: Significando o protocolo de úlcera por pressão como instrumento de qualificação para o cuidado gerencial do enfermeiro, 2015, Santa Maria ${ }^{(11)}$. 
mas de outros processos da assistência, não é só o cuidado intuitivo que se tinha antes, mas um cuidado sistematizado que tem agora, através do uso de protocolos de LP, então se avalia o paciente e se dá continuidade no processo nos outros plantões. (E7)

Relaciono o protocolo com qualidade do cuidado, segurança, uniformização. Eu acho que permite todo o embasamento legal, tu não faz as coisas da tua cabeça, está ali determinado, para tua conduta, para ter uma base do que fazer, isto só vai melhorar a qualidade porque vai ter uma uniformidade, uma continuidade do cuidado e para tu fazeres uma avaliação depois. (E18)

O protocolo é uma inovação, abre os horizontes, traz conhecimentos de como tratar o paciente com LP. (E9)

Outro aspecto importante revelado pelos profissionais nesta subcategoria é que eles percebiam o protocolo como um instrumento de atualização do conhecimento e de aperfeiçoamento das práticas de cuidado em saúde, facilitando a tomada de decisão, como pode-se notar nos seguintes trechos:

O protocolo serve para me orientar, me guiar, para eu falar a mesma linguagem dos outros, para, no caso de dúvidas, eu ter onde recorrer e para me atualizar também. Por exemplo, esses dias uma colega estava com dúvidas, eu disse para ela ver o protocolo. (E9)

Para tomar decisões tu precisas de conhecimento e o protocolo te dá este conhecimento. Como vêm profissionais novos, tu não precisas dizer como fazer, tu orientas a leitura do protocolo. O protocolo está bem explicado, bem pontuado, o novo profissional que chega consegue visualizar e ver como é feito aqui, como se previne LP, está no protocolo, todos os passos, tudo o que precisa fazer, tem a literatura ali. (E14)

\section{CONSIDERANDO O PROTOCOLO UMA FERRA- MENTA DE ENSINO}

Os enfermeiros reconhecem a importância do acadêmico de enfermagem no contexto hospitalar, logo, consideraram o protocolo uma ferramenta de ensino, que deve ser utilizada para orientar e qualificar as atividades práticas desenvolvidas pelos alunos no hospital, fortalecendo seus conhecimentos.

Temos alunos da Federal, mas também de outras universidades. Então, deveria ter um planejamento para que estes alunos fossem orientados sobre os protocolos. O objetivo seria instrumentalizar para a vida profissional, porque a gente sabe que, lá fora, a história é outra, a realidade é outra. (E3)

Eu também peço bastante para os alunos da enfermagem acessarem o protocolo, eu estimulo, utilizo o protocolo como uma ferramenta de ensino. Eles entram com muita vontade de conhecer as coberturas, então eu sempre apresento o protocolo para eles olharem, até como uma forma de estudo. Eles ficam bem interessados, 
gostam; eu gosto muito porque está bem explicado. (E9)
PERCEBENDO O PROTOCOLO COMO INSTRUMENTO DE EDUCAÇÃO EM SERVIÇO

A implantação e a manutenção, incluídas as fases de aplicação e avaliação, de novos processos a serem utilizados na prática dos profissionais de saúde, destacando-se os protocolos de enfermagem, devem estar organizadas numa perspectiva de sensibilizá-los constante.

Nesse sentido, ações devem ser sistematizadas para que se consiga a incorporação do emprego do protocolo no cotidiano de trabaIho do profissional enfermeiro. Para tanto, são necessárias atividades contínuas de educação permanente, com o objetivo de manter os profissionais atualizados para a qualificação do cuidado e, ainda, sensibilizados para a permanência dessas ações.

De modo geral, os enfermeiros expressaram, em suas falas, que o conhecimento adquirido a partir dos protocolos qualifica $o$ cuidado e que é preciso estratégias que os mantenham estimulados para sua utilização na gerência do cuidado. Assim, a educação permanente é importante para a manutenção dos conhecimentos dos profissionais nas suas práticas diárias.

Eu acho que deveria ser mais reforçada esta questão do protocolo, porque a gente tem o protocolo, e acho que, de vez em quando, deve ser retomado isto, porque, por mais que a gente faça um treinamento, a cada seis meses deve ser reforçado. Educação permanente. Passar nas unidades. Eu acho que o grupo de lesões já fez isto, acho que tem que vir passar em um turno, depois em outro, e mostrar o protocolo na Internet, chamar o pessoal e mostrar. (E1)

Tudo que é inserido de novo é difícil de implementar, mas acredito que chamar o pessoal, fazer treinamento no dia a dia, durante o plantão, na unidade, vir ali conversar, demonstrar, relembrar, porque senão acaba caindo na rotina, caindo no esquecimento. Então, eu acho importante, sobretudo agora que a gente vai receber muitos colegas novos, que o pessoal viesse trazer informações. (E11)

Eu acho que o que falta em todas as instituições é o tempo para atualização, para o estudo. Eu acho que o protocolo precisa ser trabalhado mensalmente, diariamente. (E12)

Quando a gente começou com os treinamentos, nós apreendemos um pouco mais, a gente começou a ver que existe uma série de questões que envolvem o surgimento da úlcera e aquilo me deu alívio grande, porque me mostrou que existem outros fatores que levam à lesão, não era só a má vontade, que não era só a mudança de decúbito, que não era só a umidade. Acho que melhorou com o protocolo porque começou a se falar mais. (E20)

\section{EVIDENCIANDO A IMPORTÂNCIA DOS PROTO- COLOS NA SEGURANÇA DO PACIENTE}

A segurança do paciente e dos profissionais no cuidado dispensado na prevenção e tratamento de LP, a partir da utilização do protocolo, 
foi descrita pelos enfermeiros como uma atividade que agrega qualidade ao cuidado, uma vez que orienta as ações. Eles perceberam que o uso dos protocolos garante maior probabilidade de uma assistência segura e qualificada.

O protocolo serve para padronizar nossas ações, para que todos utilizem a mesma linguagem. Para mim, não só o protocolo de LP, todos os outros protocolos eu gosto de seguir, porque é muito importante para padronizar para segurança do paciente. Para mim, serve para isto: tentar falar a mesma linguagem, todos os enfermeiros de todos os setores. (E9)

Trabalhar com protocolo significa mais segurança porque tenho algo escrito, orientando a mim e os demais funcionários que a gente tem na unidade. (E11)

O protocolo nos dá segurança em tomar decisões, de olhar uma ferida e tomar uma decisão, qual o melhor tratamento para aquela ferida. Segurança para o profissional e para o paciente, com certeza, está totalmente ligado, porque, se existe um protocolo e tu o segues, tu tens o conhecimento, tu estás realizando um cuidado seguro para o paciente e para evitar LP. Um dos itens sobre segurança do paciente é evitar as lesões, então a gente precisa ter o cuidado. (E14)

\section{DISCUSSÃO}

O estudo evidenciou que os enfermeiros percebem o protocolo de prevenção e trata- mento de LP como uma ferramenta de qualificação profissional, de ensino, de educação em serviço, de avaliação e de fundamental importância para a segurança do paciente. Nesse sentido, tem-se como consequência de sua implementação, a qualificação profissional da equipe de enfermagem para o gerenciamento do cuidado em LP.

A partir do exposto, pode-se afirmar que o uso de protocolos pelos enfermeiros é uma ferramenta gerencial que promove a qualidade da assistência prestada e a segurança do paciente ${ }^{(12,13)}$. Acredita-se que assegurar e garantir o gerenciamento do cuidado, utilizando-se de protocolos baseados em evidências científicas e mediados pela qualidade, são objetivos importantes para as instituições de saúde que buscam a excelência de seus serviços ${ }^{(14)}$.

Vale destacar que o enfermeiro, na atribuição de suas funções, necessita de conhecimento científico adequado e atualizado, de habilidade prática, de competências clínicas e gerenciais, dentre outras características relevantes ao exercício da profissão ${ }^{(13,15,16)}$.

Logo, as instituições de saúde, devem prezar pela qualidade dos serviços prestados, considerado como fator necessário para a competitividade no mercado de trabalho, para isso é preciso educação permanente com vistas à qualificação profissional e ao aprimoramento do serviço prestado(17).

Os enfermeiros têm empregado, nas suas atividades gerenciais, elevados padrões éticos e profissionais de cuidado e tratamento, com o objetivo de manter a qualidade e a eficiência ${ }^{(18)}$. Os participantes deste estudo destacaram em seus depoimentos a interação entre qualidade da assistência e segurança do paciente como temáticas que devem permear o cuidado. É importante destacar que a incidência de LP tem sido vista como um dos 
principais indicadores para a avaliação da qualidade da assistência de enfermagem ${ }^{(19)}$.

Os enfermeiros também demonstraram compreender o protocolo como uma ferramenta de atualização do conhecimento, que não pode ser reduzida apenas a um instrumento simplista e, sim, que estabelece relações e interações dinâmicas e complexas que qualificam o cuidado, por meio da constante revisão, avaliação e atualização.

A completude percebida pelos enfermeiros com relação a esse instrumento se destaca quando o gerenciamento do cuidado por meio dele vai além do cuidado clínico, sendo visto, também, como prática de educação em serviço e como instrumento de ensino. Em relação à aquisição das competências clínicas, estudo ${ }^{(15)}$ demonstrou que a aprendizagem se dá principalmente no contexto da prática(15), destacando-se o uso dos protocolos como instrumento para a aquisição e desenvolvimento dessas competências.

Nesse sentido, destaca-se o quão importante se fazem as atividades de educação permanente que utilizem os protocolos como instrumento facilitador do processo, uma vez que possibilitam a atualização de conhecimento da equipe de enfermagem e dos acadêmicos de enfermagem que realizam seus estágios práticos em cenários análogos ao estudado. $\mathrm{O}$ acadêmico de enfermagem, inserido no contexto hospitalar, é impulsionado a exercer e a amadurecer o seu papel profissional ao articular o conhecimento teórico com a prática. Além disso, as atividades de educação permanente facilitam o gerenciamento das unidades de cuidado, uma vez que o enfermeiro é o facilitador dessas ações ${ }^{(14)}$.

As limitações deste estudo estão relacionadas ao contexto onde foi desenvolvida a pesquisa, uma vez que a gerência de enfermagem baseada em protocolos é uma prática nova na instituição.

\section{CONCLUSÃO}

Nesta pesquisa, a temática possibilitou um espaço para que os enfermeiros refletissem sobre o protocolo de prevenção e tratamento de LP e as implicações de seu uso na prática diária, destacando ser uma ferramenta do gerenciamento do cuidado que permite atualização, ensino e reflexão do processo de trabalho com vistas à qualificação.

Conclui-se que os enfermeiros significaram a relação enfermeiro-paciente-protocolo em sua aplicação clínica, revelando uma complexa rede de interações que integram o fazer cotidiano do enfermeiro, o gerenciamento do cuidado, a educação em serviço e no ensino e, principalmente, a garantia de segurança e qualidade do cuidado ao paciente.

\section{REFERÊNCIAS}

1. Mororó DD, Enders BC, Lira AL, Braz da Silva CM, Menezes RM Concept analysis of nursing care management in the hospital context. Acta Paul Enferm. 2017; 30(3):323-32.

2. Lorenzetti J, Oro J, Matos E, Gelbcke FL. Work organization in hospital nursing: literature review approach. Texto \& contexto enferm. [Internet]. 2014 [Cited 2016 maio 05];23(4):1 104-12. Available from: http://www.scielo.br/pdf/tce/ v23n4/0104-0707-tce-23-04-01104.pdf

3. Soares RSA, Lima SBS, Kessler M, Eberhardt TD, Rorato ARS, Dias CC. Coding and analyzing data from the perspective of the theory based on data: case report. J Nurs UFPE on line. [Internet]. 2015 [Cited 2016 maio 1];9(8):8919-22. Available from:http://www.revista.ufpe.br/revistaenfermagem/index.php/revista/article/view/7894

4. Santos JLG, Pestana AL, Guerrero P, Meirelles BSH, Erdmann ALI. Práticas de enfermeiros na gerência do cuidado em enfermagem e saúde: revisão integrativa. Rev bras enferm. [Internet]. 2013 [cited 2016 abr 10]; 66(2):257-63. Avai- 
lable from: http://www.scielo.br/pdf/reben/ v66n2/16.pdf.

5. Senna MH, Drago LC, Kirchner AR, Santos JL, Erdmann AL, Andrade SR. Meanings of care management built throughout nurses' professional education. Rev Rene. 2014; 15(2):196-205.

6. Paes GO, Mello ECP, Leite JL, Mesquita MGR, Oliveira FT, Carvalho SM. Care protocol for clients with respiratory disorder: tool for decision making in nursing. Esc Anna Nery Rev Enferm. 2014;18(2):303-10.

7. Amir Y, Lohrmann C, Halfens RJ, Schols JM. Pressure ulcers in four Indonesian hospitals: prevalence, patient characteristics, ulcer characteristics, prevention and treatment. Int Wound J. [Internet]. 2017[29 cited mai 2018];14(1):184193. Available from: https://onlinelibrary.wiley. com/doi/epdf/10.1111/iwj.12580

8. National Pressure Ulcer Advisory Panel. National Pressure Ulcer Advisory Panel (NPUAP) announces a change in terminology from pressure ulcer to pressure injury and updates the stages of pressure injury [Internet]. 2016 [cited 2016 Out 9]. Available from: http://www.npuap. org/national-pressure-ulcer-advisory-panel-npuap-announces-a-change-in-terminology-from-pressure-ulcer-to-pressure-injury-and-updates-the-stages-of-pressure-injury/

9. Soares RSA, Lima SBS, Eberhardt TD, Kessler $M$, Fonseca GGP, Saul AMR et al. Protocolos de prevenção e tratamento de úlceras por pressão: análise da produção brasileira. Saúde (Santa Maria). 2016;42:63-72.

10. Corbin J, Strauss A. Basics of qualitative research: techniques and procedures for developing Grounded Theory. 4th ed. Los Angeles (CA): SAGE; 2015

11. Soares RSA. Significando o protocolo de úlcera por pressão como instrumento de qualificação para o cuidado gerencial do enfermeiro. [dissertação]. Santa Maria (RS): Universidade Federal de Santa Maria; 2015.

12. Sales $C B$, Bernardes $A$, Gabriel CS, Brito MFP, Moura AA, Zanetti ACB. Standard Operational Protocols in professional nursing practice: use, weaknesses and potentialities. Rev Bras Enferm [Internet]. 2018 Fev [cited 2018 May 28];71(1):126-34. Available from: http://www. scielo.br/pdf/reben/v71n1/0034-7167-reben-71-01-0126.pdf

13. Siman AG, Brito MJM. Changes in nursing practice to improve patient safety. Rev Gaúcha Enferm [Internet]. 2016 [cited 2018 May 28];37(spe):e68271. Available from: http://www. scielo.br/pdf/rgenf/v37nspe/en_0102-6933rgenf-1983-14472016esp68271.pdf

14. Moura EC, Luz MHBA, Cavalcante TB, Queiroz AAFLN, Furtado LB, Monte BKS. Updating pf the assistance protocol for pressure ulcer prevention: evidence based practice. Rev enferm UFPE on line [Internet]. 2016 [cited 2018 May 29];10(Supl):1498-506. Available from: https:// periodicos.ufpe.br/revistas/revistaenfermagem/article/view/11091/12542

15. Aued GK, Bernardino E, Peres AM, Lacerda MR, Dallaire C, Ribas EN. Clinical competences of nursing assistants: a strategy for people management. Rev Bras Enferm. [Internet]. 2016 Feb [cited 2018 May 29];69(1):142-9. Available from: http://www.scielo.br/pdf/reben/v69n1/ en_0034-7167-reben-69-01-0142.pdf

16. Melnyk BM, Gallagher-Ford L, Long LE, Fineout-Overholt E. The establishment of evidence-based practice competencies for practicing registered nurses and advanced practice nurses in real-world clinical settings: proficiencies to improve healthcare quality, reliability, patient outcomes, and costs. Worldviews Evid Based Nurs. 2014 Feb;11(1):5-15.

17. Velho JM, Treviso P. Implantação de programa de qualidade e acreditação: contribuições para a segurança do paciente e do trabalhador. Rev adm saúde [Internet]. 2013 Jul-Set [cited 2018 May 29];15(60):90-4. Available from: http:// cqh.org.br/portal/pag/anexos/baixar.php?p_ ndoc $=935 \& p \_n a n e x o=480$

18. Orvik A, Vagen SR, Axelsson SB, Axelsson R. Quality, efficiency and integrity: value squeezes in management of hospital wards. J nurs manag. 2015;23:65-74.

19. Pinto VRS, Ferreira SCM. Indicators for the assessment of the quality of nursing care: a descriptive-exploratory study. Online braz j nurs [internet]. 2017 Mar [cited 2018 May 29];16(1):140-51. Available from: http://www.objnursing.uff.br/ index.php/nursing/article/view/5481 
20. Sade PMC, Peres AM. Development of nursing management competencies: guidelines for continuous education services. Rev Esc Enferm USP. [internet] 2015 [cited 2016 May 20];49(6):9918. Available from: http://www.scielo.br/pdf/ reeusp/v49n6/0080-6234-reeusp-49-06-0991. pdf
Todos os autores participaram das fases dessa publicação em uma ou mais etapas a seguir, de acordo com as recomendações do International Committe of Medical Journal Editors (ICMJE, 2013): (a) participação substancial na concepção ou confecção do manuscrito ou da coleta, análise ou interpretação dos dados; (b) elaboração do trabalho ou realização de revisão crítica do conteúdo intelectual; (c) aprovação da versão submetida. Todos os autores declaram para os devidos fins que são de suas responsabilidades o conteúdo relacionado a todos os aspectos do manuscrito submetido ao OBJN. Garantem que as questões relacionadas com a exatidão ou integridade de qualquer parte do artigo foram devidamente investigadas e resolvidas. Eximindo, portanto o OBJN de qualquer participação solidária em eventuais imbróglios sobre a matéria em apreço. Todos os autores declaram que não possuem conflito de interesses, seja de ordem financeira ou de relacionamento, que influencie a redação e/ou interpretação dos achados. Essa declaração foi assinada digitalmente por todos os autores conforme recomendação do ICMJE, cujo modelo está disponível em http://www.objnursing.uff.br/normas/ DUDE_final_13-06-2013.pdf

Recebido: $22 / 08 / 2016$

Revisado: 28/05/2018

Aprovado: 28/05/2018 